Received: 2017.04.24 Accepted: 2017.05.11 Published: 2017.06.26

\section{Gastrointestinal Stromal Tumor Diagnosed During Donor Procurement: The Experience of a Single Institution and Review of the Literature}

1 Institute of Histopathology and Molecular Diagnosis, Careggi Hospital, Florence, Italy

2 Department of Experimental and Clinical Medicine, University of Florence, Florence, Italy

3 Pathology Unit, A. Meyer Children's University Hospital, Florence, Italy

4 Regional Center Allocation Organs and Tissues, Careggi Hospital, Florence, Italy
Corresponding Author: Source of support:

Background: Gastrointestinal stromal tumors (GISTs) are rare neoplasms accounting for 5\% of all sarcomas, but they are the most common mesenchymal tumors within the gastrointestinal (GI) tract. GISTs occur throughout the GI tract, with the stomach being the most frequent site of origin.

Material/Methods Herein, we describe 5 cases of GISTs diagnosed during donor procurement. Frozen sections of the tumors were highly suspicious for GISTs, and mitotic count evaluation was also performed. However, the definitive diagnosis and risk assessment required immunohistochemical staining for CD117 and DOG-1, together with the mitotic count performed on permanent sections.

Results: $\quad$ From our 5 donors, 2 kidneys and 1 liver were transplanted with no evidence of donor-transmitted neoplasia after 18 and 46 months, respectively.

Conclusions: We describe 5 cases of GISTs with no risk of progressive disease. In accordance with the latest guidelines of the European Committee on Organ Transplantation, only donors with small gastric GIST were accepted for liver and renal transplant.

MeSH Keywords: $\quad$ Gastrointestinal Stromal Tumors • Kidney Transplantation • Liver

Abbreviations: $\quad$ GIST - gastrointestinal stromal tumor; CRAOT - Tuscany Regional Transplant Center; CNT - National Transplant Center; GI - gastrointestinal; HPFs - high-power fields

Full-text PDF: http://medscitechnol.com/abstract/index/idArt/905005

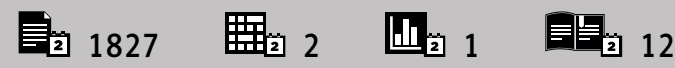




\section{Background}

Gastrointestinal stromal tumors (GISTs) are the most common primary mesenchymal neoplasm of the GI tract. GISTs are most commonly seen in the stomach $(60 \%)$, small intestine (30\%), duodenum ( $5 \%)$, and colorectum $(<5 \%)$, and rarely in the esophagus $(<1 \%)[1,2]$. Recent population-based studies [3] found incidences of approximately 10 to 20 cases/million/year. These findings would translate into an annual incidence in Europe of about 8000-9000 cases and in the USA of about 4000-5000 cases. The prevalence of GISTs is much higher since many patients live with the disease for many years or develop small lesions detected during endoscopy, surgery, or radiological studies performed for other causes or at autopsy [3]. Indeed, GISTs vary from diminutive incidental findings to symptomatic tumors that most commonly reveal themselves by occult or visible gastrointestinal bleeding and less commonly by obstruction, perforation, or palpable mass. Moreover, it has been reported that about $10 \%$ of GISTs occur in patients harboring other different solid tumors and rare cases have been described in immunocompromised patients [1-3]. The median age is around $60-65$ years and several series have found a slight predominance of males [3]. Pediatric GISTs are very rare and represent a distinct subset with specific clinical-pathological and molecular findings [3]. Most GISTs are sporadic lesions but they may also be linked to syndromes such as Carney triad syndrome, Carney-Stratakis syndrome, and neurofibromatosis type 1. Rare familial GISTs have also been well described [3].

GISTs are thought to arise from the interstitial cells of Cajal or, most probably, from their stem cell-like precursor. Most GISTs harbor activating mutations of either KIT (about $75 \%$ of cases) or PDGFRA (in about $10 \%$ of cases). BRAF mutations have been reported to occur very rarely [3]. KIT/PDGFRA wild-type GISTs may also occur, especially in the setting of syndromic GISTs or pediatric GISTs [3]. More than $90 \%$ of GISTs are characterized by the immunohistochemical expression of CD117 and/or DOG-1; a smaller subset of GISTs, more frequently gastric GISTs, may be CD34-positive [1-3]. On gross examination, GISTs present as well-circumscribed, highly vascular tumors. On cut-surface they may appear fleshy pink or tan-white tumors and may show lobular appearance, central cystic degeneration, or hemorrhagic foci, depending on their dimensions [1,3]. Histologically, most (approximately 70\%) GISTs have spindle cell morphology, and less frequently have epithelioid cell morphology (approximately 20\%) and mixed spindle-epithelioid cell morphology (approximately 10\%) [1,3]. As regards differential diagnosis, smooth-muscle tumors, nerve sheath tumors, desmoids, inflammatory myofibroblastic tumors, inflammatory fibroid polyps, and undifferentiated sarcomas are the tumors most commonly confused with GISTs [1-3]. These tumors have been mostly reported as CD117- and DOG-1negative and the majority have distinct immunohistochemical markers not expressed in GISTs. Risk stratification of primary GISTs is based on tumor size, mitotic index, and anatomic location [1]. Mitotic index is determined by evaluating 50 highpower fields (HPFs). This scheme identifies 5 different categories that help to predict the risk of aggressive clinical behavior (expressed as none, very low, low, moderate, and high) [1]. In recent years, additional risk schemes have been proposed [4]. In particular, the presence of tumor rupture before or during surgery has been demonstrated to be a highly unfavorable prognostic factor and its occurrence offsets the conventional prognostic parameters [5].

The data from the literature report few cases of GISTs occurring in transplant recipients arising de novo after immunosuppressive therapy [6-8]. To date, only 1 case of gastric GIST during donor procurement has been reported [9]. We report 5 cases of GISTs diagnosed in the same institution during donor procurement.

\section{Material and Methods}

Cases were retrieved from the files of the Institute of Histopathology and Molecular Diagnosis, University of Florence, Careggi Hospital, Florence, Italy. Five GISTs were identified out of 847 biopsies analyzed during donor procurement from 2011 to 2016. All candidate subjects were selected as donors by the Regional Center Allocation Organs and Tissues (CRAOT) and National Transplant Center (CNT). No history of cancer was known before the donor procurement. In all cases, ultrasound abdomen examination and clinical-serological studies were negative for tumor pathology. Tissue specimens were first examined on frozen section and subsequently fixed in $10 \%$ buffered neutral formalin and were paraffin embedded. We stained $5-\mu m$ sections with hematoxylin and eosin (H\&E). Additional sections of the most representative paraffin embedded blocks were mounted on electrostatic slides and used for the immunohistochemistry. Immunohistochemical studies were performed on a Ventana BenchMark ULTRA immunostainer (Ventana Medical Systems, Tucson, AZ). The primary antibodies were: CD117 (c-kit rabbit monoclonal antibody, clone YR145, Cell Marque, Rocklin, CA), DOG1 (rabbit monoclonal antibody, clone SP31, Ventana Medical Systems), anti-Ki67 (rabbit monoclonal antibody, clone 30-9, Ventana Medical Systems), smooth-muscle actin (mouse monoclonal antibody, clone 1A4, Ventana Medical Systems), and S-100 protein (mouse monoclonal antibody, clone 4C4.9, Ventana Medical Systems). The Ventana staining procedure included pretreatment with cell conditioner 1 , followed by incubation with the antibody. The signal for antibodies was then developed with use of the UltraView Universal DAB Detection Kit. After the staining run was complete, the tissue sections were counterstained with hematoxylin. Positive controls included 
Table 1. Clinical-pathologic characters of GISTs diagnosed during donor procurement.

\begin{tabular}{|c|c|c|c|c|c|c|c|}
\hline Case & Sex & $\begin{array}{c}\text { Age } \\
\text { (years) }\end{array}$ & Site & $\begin{array}{l}\text { Diameter } \\
\text { (cm) }\end{array}$ & $\begin{array}{l}\text { Mitosis } \\
\text { (50 HPFs) }\end{array}$ & Transplanted organs & Follow-up \\
\hline 1 & $\mathrm{~F}$ & 82 & Ileum & 1 & 0 & No & \\
\hline 2 & M & 89 & Stomach & 1.5 & 1 & No & \\
\hline 3 & $F$ & 66 & Stomach & 0.8 & 0 & Kidneys & $\begin{array}{c}\text { Two recipients } \\
\text { FOD at } 19 \\
\text { months }\end{array}$ \\
\hline 4 & $\mathrm{~F}$ & 65 & Colon & 0.8 & 0 & No & \\
\hline 5 & $M$ & 74 & Stomach & 0.5 & 0 & Liver & $\begin{array}{c}\text { FOD at } 46 \\
\text { months }\end{array}$ \\
\hline
\end{tabular}

F - Female; M - Male; HPFs - high-power fields; FOD - free of disease.

breast for CD117 and S-100 protein, appendix for actin smoothmuscle, GIST for DOG1, and skin for Ki67. Negative controls were performed by negative control mouse monoclonal antibody clone MOPC-211 and rabbit monoclonal negative control Ig (Ventana Medical Systems). Control sections were treated at the same time as sample sections. All sections were dehydrated and mounted with Permount.

The diagnosis of GIST was made according to the morphological and immunohistochemical criteria of Miettinen et al. [1]. Mitotic activity was evaluated on 50 consecutive high-power fields (HPFs). Clinical-pathological studies and follow-up data were provided by the CRAOT.

\section{Results}

Clinical-pathological data of our series are shown in Table 1. Regarding sex distribution, 3 (60\%) of 5 donors were female, median age was 74 years, and the average diameter of the lesions was $0.9 \mathrm{~cm}$. Within our 5 cases, the tumor was located in the stomach in $3(60 \%)$ cases, in the ileum in $1(20 \%)$ case, and in the colon in $1(20 \%)$ case. Histologically, all cases had spindle cell morphology (Figure 1A, 1B) on both frozen and definitive sections. One gastric case showed a mitotic count of 1/50 HPFs on frozen sections subsequently confirmed on definitive sections; all the other cases had no mitosis. All cases were positive for CD117 and DOG-1 (Figure 1C, 1D). No immunoreactivity was found for smooth-muscle actin, S-100 protein, and CD34. According to Miettinen et al. [1,4], in all cases there was no risk of progressive disease. Two kidneys from the same donor and the liver from another donor were transplanted in 3 different receivers with no evidence of donor-transmitted neoplasia after 18 and 46 months, respectively.

\section{Discussion}

Although cadaveric donation has increased in recent years, it does not satisfy the demand for transplantation, for which increasing numbers of patients are awaiting. Expanding the organ donor pool is a high priority. In the meantime, acceptable organ quality must be guaranteed without exposing recipients to unacceptable risks. In the presence of tumor pathology in donors, it is mandatory to identify the histological type and grade to perform transplantation with a low or no risk of disease transmission [4,9-11].

Cancer in transplant recipients can be classified into 4 categories: 1) donor-transmitted, which is present at the time of transplantation; 2) donor-derived, which develops within the donor cells after transplantation; 3) de novo, which develops from the recipient cells; and 4) recurrent cancer, which is the recurrence of a cancer after transplantation, that the recipient had before transplantation [11,12]. In the latest guidelines for the quality and safety of organs for transplantation, the European Committee on Organ Transplantation [10] set guidelines for GISTs during donor procurement. Small GISTs, diagnosed during procurement, less than $2 \mathrm{~cm}$ in size, with a mitotic index $\leq 5 / 50$ HPFs, located in the stomach or in the duodenum may be acceptable for organ donation with a lowto-intermediate risk of transmission [10]. GISTs of other sites or of larger size and higher mitotic count are associated with an increased risk of metastases and a high risk of transmission [10]. Mitotic count evaluation and immunohistochemical stains for CD117 and DOG1 must be performed in permanent sections [10].

To the best of our knowledge, 5 cases of de-novo GISTs after kidney transplantation have been reported in the English literature [6-8]. Table 2 summarizes the main clinical-pathologic characters. Occurrence of GISTs in recipients was first reported in 2 patients by Agaimy et al. [6]. They described 2 female 


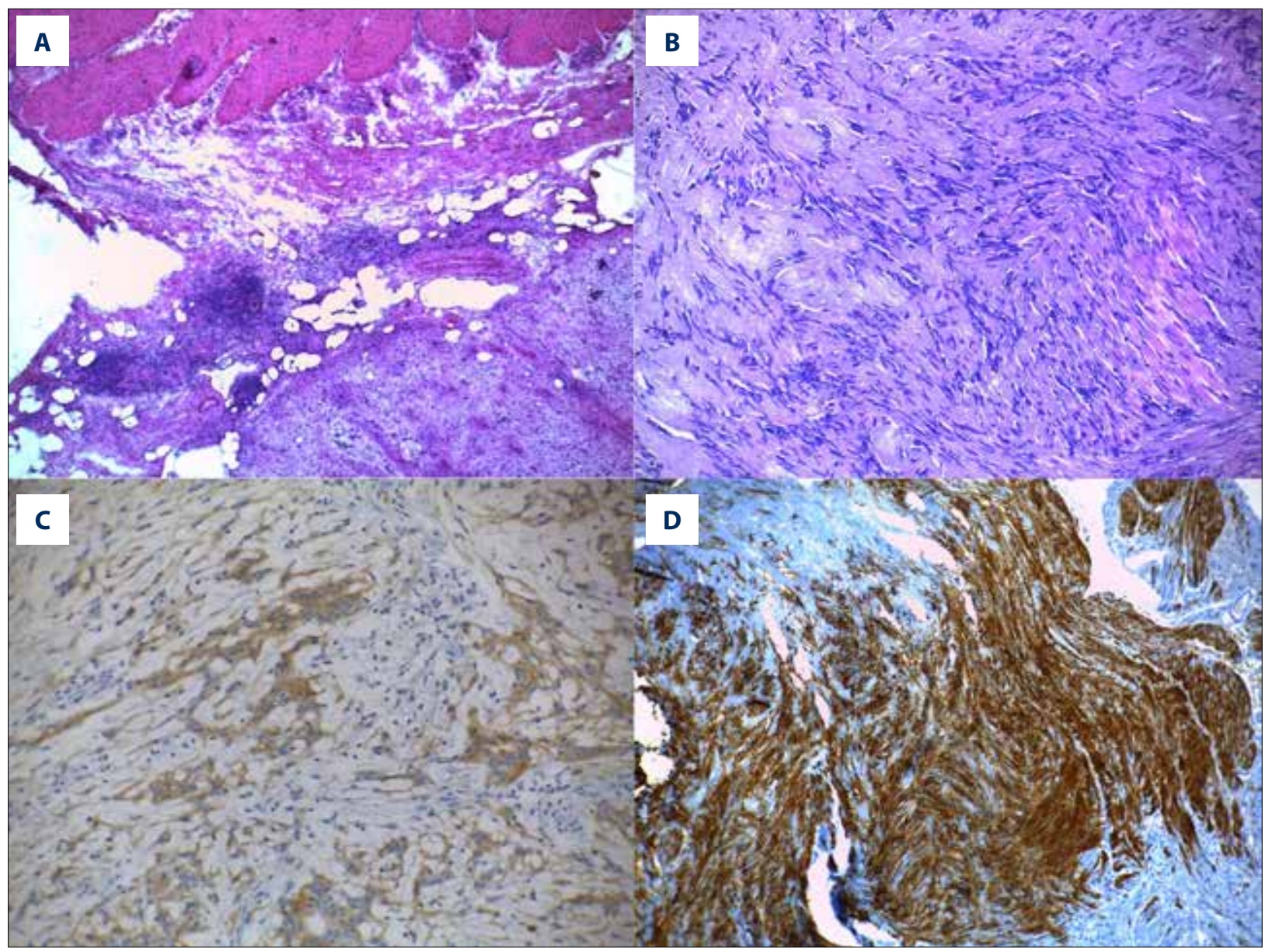

Figure 1. (A). Low-magnification view of intestinal GIST. (B) The high-magnification view of the tumor shows sclerosing appearance with collagen deposition and moderate cellularity. (C) Cytoplasmic CD117-staining. (D) DOG1 membrane staining.

Table 2. De novo GISTs in recipients: literature review.

\begin{tabular}{|c|c|c|c|c|c|c|c|}
\hline Reference & Sex & $\begin{array}{c}\text { Age } \\
\text { (years) }\end{array}$ & Site & $\begin{array}{l}\text { Diameter } \\
\text { (cm) }\end{array}$ & $\begin{array}{l}\text { Mitosis } \\
\text { (50 HPFs) }\end{array}$ & $\begin{array}{l}\text { Years post- } \\
\text { transplant }\end{array}$ & Follow-up \\
\hline $\begin{array}{l}\text { Agaimy } \\
\text { et al. [6] }\end{array}$ & $\mathrm{F}$ & 59 & Stomach & 3,5 & $<5$ & 40 months & $\begin{array}{l}\text { FOD at } 68 \\
\text { months }\end{array}$ \\
\hline $\begin{array}{l}\text { Agaimy } \\
\text { et al. [6] }\end{array}$ & $\mathrm{F}$ & 58 & Small bowel & 23 & 14 & 8 years & NA \\
\hline Mulder [7] & M & 72 & Stomach & 5 & $>10$ & 21 years & $\begin{array}{c}\text { Died after } 4 \\
\text { years }\end{array}$ \\
\hline $\begin{array}{l}\text { Cheung } \\
\text { et al. [8] }\end{array}$ & $M$ & 64 & Stomach & 3 & 9 & 2 years & $\begin{array}{c}\text { DOD after } 2 \\
\text { years }\end{array}$ \\
\hline $\begin{array}{l}\text { Cheung } \\
\text { et al. [8] }\end{array}$ & $M$ & 48 & $\begin{array}{l}\text { Multiple } \\
\text { abdominal } \\
\text { masses }\end{array}$ & NA & NA & 1 years & $\begin{array}{l}\text { Target therapy } \\
\text { alive at } 10 \text { years }\end{array}$ \\
\hline
\end{tabular}

F - Female; M - Male; DOD - died of disease; NA - not available; FOD - free of disease.

patients aged 59 and 58 years. Both patients had undergone renal transplantation. The first patient developed a low-risk epithelioid gastric GIST $(3.5 \mathrm{~cm}$ in size and $<5$ mitoses $/ 50 \mathrm{HPFs})$
40 months after transplantation; the second patient presented a high-risk ruptured spindle cells GIST of the small bowel (23 cm in size and 14 mitoses/50 HPFs) 96 months after 
transplantation. Mutational studies for KIT or PDGFRA were not performed and the treatment modality was not reported. Mulder et al. [7] described the third case, a 72-year-old man who developed a high-risk gastric GIST $(5 \mathrm{~cm}$ in size with $>10$ mitosis/50 HPFs) 21 years after renal transplantation. He underwent emergency surgical resection and 2 years later developed multiple peritoneal metastasis. The patient was treated with imatinib associated with cyclosporine but required decreasing doses of both drugs for a decline in renal function. They stress the importance of the interaction between imatinib and cyclosporine. Molecular studies were not performed. Cheung et al. [8] reported 2 other cases: 2 male patients aged 64 and 48 years. The first patient developed a moderate-risk epithelioid gastric GIST ( $3 \mathrm{~cm}$ in size and 9 mitosis/50 HPFs) 24 months after renal transplantation. He refused adjuvant imatinib therapy. After 2 years, liver metastasis was shown but the patient died of multidrug-resistant pneumonia. The second patient presented with multiple mesenteric masses, diagnosed as spindle cell GIST, 12 months after renal transplantation. The patient was treated first with imatinib and subsequently with sirolimus. Serial imaging confirmed complete resolution of tumors after a follow-up period of more than 10 years. In summary, the data from the literature are very few and differ in

\section{References:}

1. Miettinen M, Lasota J: Gastrointestinal stromal tumors. Review on morphology, molecular pathology, prognosis, and differential diagnosis. Arch Pathol Lab Med, 2006; 130: 1466-87

2. Miettinen M, Lasota J: Gatrointestinal stromal tumors: Pathology and prognosis at different sites. Semin Diagn Pathol, 2006; 23(2): 70-83

3. Liegl-Atzwanger B, Fletcher JA, Fletcher CDM: Gastrointestinal stromal tumor. Virch Arch, 2010; 456: 111-27

4. The ESMO/European Sarcoma Network Working Group: Gastrointestinal stromal tumor: ESMO clinical practice guidelines for diagnosis, treatment an follow-up. Annals Once, 2012; 23(7); vii49-55

5. Joensuu H, Vehtari A, Riihimaki J et al: Risk of recurrence of gastrointestinal stromal tumor after surgery: An analysis of pooled population-based cohorts. Lancet Once, 2012; 13(3): 265-74

6. Agaimy A, Wunsch PH: Gastrointestinal stromal tumors (GIST) in kidney transplant recipients - a report of two cases. Nephrol Dial Transplant, 2007; 22: $1489-90$ clinical presentation and treatment. Unfortunately, there are no data on the molecular setting of the moderate- and highrisk GISTs reported.

Concerning the detection of GISTs during donor procurement, only 1 previous case has been reported in the English literature [9]. The authors analyzed 4654 referred donors and there were 377 potential donors with neoplasm; according to histological tumor type and grade, 28 cases were suitable for organ transplantation. One of these cases was a gastric GIST considered acceptable for liver transplantation. No other clinical and pathological data were reported.

\section{Conclusions}

We described 5 cases of GISTs with no risk of progressive disease (size $<2 \mathrm{~cm}$, mitotic index $<5 / 50 \mathrm{HPFs}$ ). In accordance with the latest guidelines of the European Committee on Organ Transplantation [10], only donors with small gastric GIST are accepted for liver and renal transplant. Since GISTs are rare tumors, multicenter studies are needed to confirm and validate current guidelines.

7. Mulder K, Egorin M, Sawyer M: Renal dysfunction in a renal transplant patient treated concurrently with cyclosporine and imatinib. Invest New Drugs, 2012; 30: 2400-2

8. Cheung CY, Lo SHK, Chan CK et al: Gastrointestinal stromal tumors in kidney transplant recipients: report of 2 cases and literature review. Asia Pac J Clin Oncol, 2017; 13(1): 104-6

9. Fiaschetti P, Protagonisti R, Stabile D et al: The use of neoplastic donors to increase the donor pool. Transplant Proc, 2012; 44: 1848-50

10. European Committee on organ transplantation. Guide to the quality and safety of organs for transplantation. 2016; $6^{\text {th }}$ edition: 182

11. Naleskin MA, Woodle ES, Di Maio JM et al: Donor-transmitted malignancies in organ transplantation: assessment of clinical risk. Am J Transpl, 2011; 11: $1140-47$

12. Al-Azzawi Y, Stein L, Shrestha R et al: Donor-derived hepatic neuroendocrine tumor: Pause before proceeding with liver retransplantation. Transplant Dir, 2016; 2(7): 1-3 